\title{
Facial Expression Recognition Using Data Mining Algorithm
}

\author{
Priyanka Tripathi M, Kesari Verma, Ligendra Kumar Verma, and Nazil Parveen
}

\begin{abstract}
Facial expression is one of the most powerful, natural, and abrupt means for human beings which have the knack to communicate emotion and regulate inter-personal behaviour. In this paper we focus on two different approaches of expression recognition. First template based method, second appearance based method i.e. principle component analysis. In template based we make use of template matching to excerpt templates of different facial components. The facial expression information is mostly concentrate on facial expression information regions, mouth, eye and eyebrow regions areas are segmented from the facial expression images. Using these templates we calculate facial characteristics points (FCP's).Then we define 30 facial characteristic points to describe the position and shape of the above three organs to find diverse parameters which are input to the decision tree for recognizing different facial expressions.
\end{abstract}

Index Terms-Decision tree, facial characteristics points extraction, PCA, feature extraction, template matching.

\section{INTRODUCTION}

Human facial expression recognition (FER) has attracted much attention in recent years because of its importance in realizing highly intelligent human-machine interfaces. Human facial expression contains extremely abundant information of human's behavior and can further reflect human's corresponding mental state. As human face plays a crucial role in interpersonal communication, facial expression analysis is active in the fields of affective computing and intelligent interaction. Several FER methods [1]-[8] have been proposed. Facial expression play important role in cognition of human emotions and facial expression recognition is the base of emotions understanding [9].

The Facial Action Coding System (FACS) developed by Paul Ekman and Wallace V. Friesen [1] is the most widely used and validated method for measuring and describing facial behavior. Ekman and Friesen defined six basic emotions (happiness, sadness, fear, disgust, surprise, and anger). Each of these six basic emotions corresponds to a unique facial expression. They defined the facial action coding system (FACS), a system developed in order to enable facial expression analysis through standardized coding of changes in facial motion. FACS consists of 46 action units (AU) which describe basic facial movements. It is based on

Manuscript received March 2, 2013; revised May 12, 2013.

Priyanka. Tripathi and Kesari Verma are with the National Institute of Technology, Raipur, India (e-mail: priyanka_tripahi@hotmail.com, keshriverma@gmail.com).

Ligendra Kumar Verma is with Raipur Institute of Technology, India (email: ligendra@rediffmail.com).

Nazil Parveen is with Central University Bilaspur India (email : 786.nazil@gmail.com) muscle activity and describes in detail the effect of each AU on face features. Hence, among the $46 \mathrm{AU}$ that shows the basic movement of face muscles, except for 5 AUs corresponding to movement of cheek, chin and wrinkles, 41 AUs are directly associated with movement of eyes, eyebrows and mouth [10]. However all 41 AUs are not necessarily required for facial characteristics points (FCP) calculation. Therefore we calculate 30 FCP's, to extract these 30 FCP's template matching mechanism is applied to match the eyes, eyebrows and mouth template. Then we define these 30 FCP's to compute the position and shape of the different components of the face, such as, eyes, eyebrows and mouth. Using these FCP's we compute the different parameters to be inputted in the decision tree algorithm for recognizing different facial expressions.

\section{Feature Extraction Using Template Matching}

Feature extraction is the process of extracting important attribute which are used to classify the images into different classes. Template matching is being carried out by making use of convolution and correlation coefficients for the highest and perfect matching. The desired eyes, eyebrows and mouth template are being excerpt from the image and the extracted results are shown in the form of bounded rectangles as in Fig.1. The algorithm work as follows.

1) Input the template and the target image.

2) Convert the images into gray scale images.

3) Find the 2-d convolution of the target and template image.

4) Find the mean and variance of the template image.

5) Form the search image from the search region of the template size.

6) Calculate the correlation score ' $\mathrm{C}$ '.

7) Find the pixel value from the search region having

8) The highest value of the correlation score ' $\mathrm{C}$ '.

9) Draw the bounding rectangles by using the index of the matched correlation score.

\section{EXtRacting THE FACIAL CHARACTERISTIC POINTS}

The bounding rectangles form around the matched template is used to deduce the value of top-left corner pixel from the rectangles. By using width and height of the template size specified in Table 1, we compute all the 30 FCP's [10]. These pixel values is used to evaluate the facial animation parameters described as opening of eyes (oe), width of eyes (we), height of eyebrows (he), opening of mouth (om), and width of mouth (wm). Table I, describes 
some of the FCP's calculations.

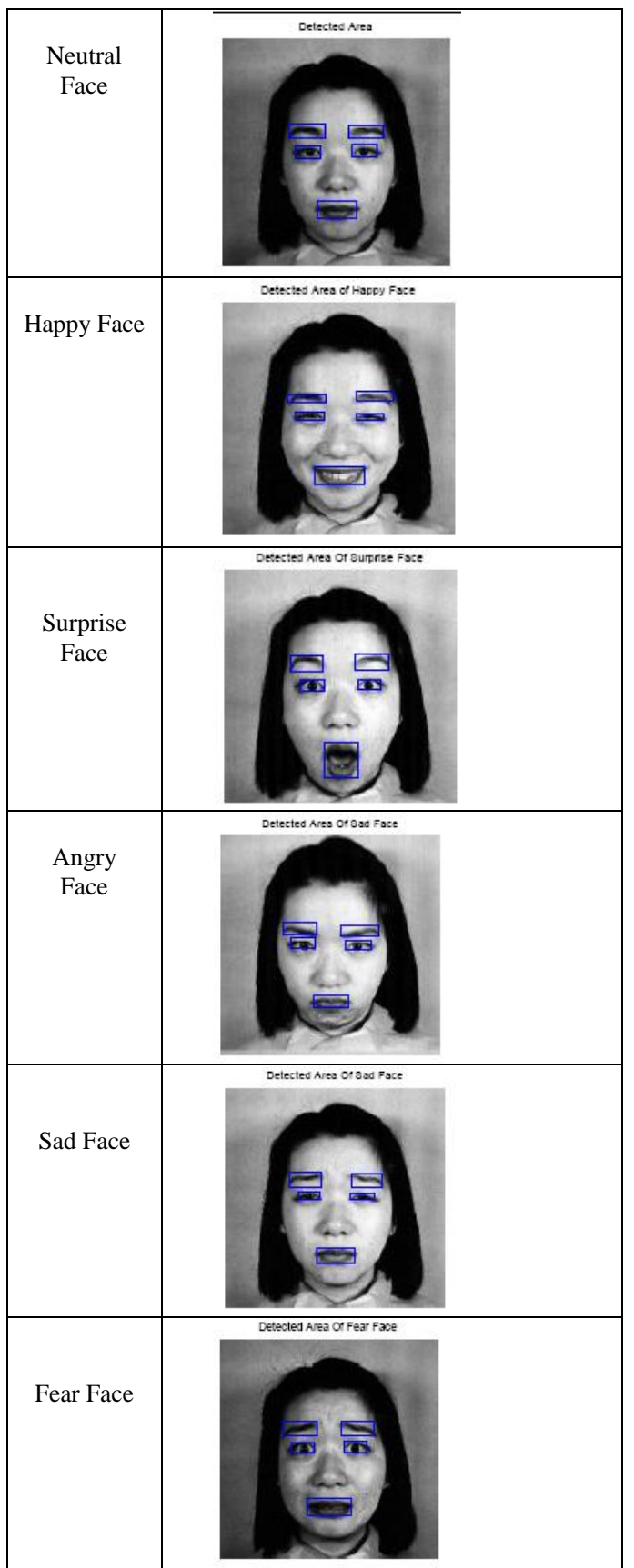

Fig. 1 Template Matching applied in different facial expressions.

TABLE I: EVALUATION OF THE FCPS

\begin{tabular}{|c|c|c|c|c|}
\hline Region & $\begin{array}{c}\text { FCP } \\
\text { Number }\end{array}$ & $\begin{array}{c}\mathrm{X} \\
\text { coordinate }\end{array}$ & $\begin{array}{c}\mathrm{Y} \\
\text { coordinate }\end{array}$ & Region \\
\hline Left eye & 1 & lle + wle & $\begin{array}{c}\text { tle }+ \\
\text { hle*4/5 }\end{array}$ & Left eye \\
\hline Right eye & 2 & lre & tre + hre $/ 2$ & Right eye \\
\hline Left eyebrow & 17 & $\begin{array}{c}1 \mathrm{lb}+ \\
\mathrm{wlb} / 2\end{array}$ & $\mathrm{tlb}+\mathrm{hlb} / 3$ & $\begin{array}{c}\text { Left } \\
\text { eyebrow }\end{array}$ \\
\hline Right eyebrow & 18 & $\begin{array}{c}\text { lrb }+ \\
\mathrm{wrb} / 2\end{array}$ & $\operatorname{trb}+\mathrm{hrb} / 2$ & $\begin{array}{c}\text { Right } \\
\text { eyebrow }\end{array}$ \\
\hline Mouth & 23 & $1 \mathrm{mo}$ & $\begin{array}{l}\mathrm{tmo}+ \\
\mathrm{hmo} / 2\end{array}$ & Mouth \\
\hline
\end{tabular}

In this way, we calculate 30 FCP's, were:

lle, lre, llb, lrb, lmo:- left of left eye, right eye, left eyebrow, right eyebrow, mouth.

wle, wre, wrb, wlb:- width of left eye, right eye, left eyebrow, right eyebrow. tle, tre, trb, tlb, tmo :- top of left eye, right eye, left eyebrow, right eyebrow, mouth.

hle, hre, hlb, hrb, hmo:- height of left eye, right eye, left eyebrow, right eyebrow, mouth.

\section{COMPUTATION OF FACIAL ANIMATION PARAMETER}

The facial animation parameters are evaluated from the FCP's in the following way [11];

Openness of eyes:

$\left(\left(\mathrm{fc} 7 \_\mathrm{y}-\mathrm{fc} 5 \_\mathrm{y}\right)+\left(\mathrm{fc} 8 \_\mathrm{y}-\mathrm{fc} 6 \_\mathrm{y}\right)\right) / 2$

Width of eyes:

$\left(\left(\mathrm{fc} 1 \_\mathrm{x}-\mathrm{fc} 3 \_\mathrm{x}\right)+\left(\mathrm{fc} 4 \_\mathrm{x}-\mathrm{fc} 2 \_\mathrm{x}\right)\right) / 2$

Height of eyebrows:

$\left(\left(f c 19 \_y-f c 1 \_y\right)+\left(f c 20 \_y-f c 2 \_y\right)\right) / 2$

Opening of mouth: (fc26_y - fc25_y)

Width of mouth: (fc24_y - fc23_y) where, fc1_x,fc2_x, fc3_x,fc4_x, fc7_y, fc5_y, fc8_y, fc6_y, are the $\mathrm{x}, \mathrm{y}$ coordinate position of the FCP's detected around the eye template. Similarly the FCP's fc1_y, fc2 $\_y$, fc19_y, fc20_y are the $\mathrm{x}, \mathrm{y}$ coordinate position detected around the eyebrow template. FCP's fc23_y, fc24_y, fc25_y and fc26_y are the $y$ coordinates of mouth template. Deriving these facial parameters from FCP's, these features can be used to detect the class_label of face data.

\section{Gini Index Classification TeChNiQue}

GINI index approach is a classification technique fall under the decision tree category. In a GINI index decision tree classification, each leaf node is assigned as a class label. The non-terminal nodes includes, the root and other internal nodes, that contain attributes test conditions to split the data set that have different characteristics. The splitting is performed based on the basis of measure of degree of impurity of the child nodes. In the GINI index the impurity of the child nodes is being calculated by using (1).

$$
\operatorname{gini}(T)=1-\sum p_{i}^{2}
$$

where pi is the relative frequency of class $\mathrm{j}$ in $\mathrm{T}$. If the split divide $\mathrm{T}$ into $\mathrm{T} 1$ and $\mathrm{T} 2$.

$$
\operatorname{gini}_{\text {spli }}(T)=\frac{n_{1}}{n} \operatorname{gini}\left(T_{1}\right)+\frac{n_{2}}{n} \operatorname{gini}\left(T_{2}\right)
$$

Using (1) we decide which attribute among the set of attribute is best for split. And by (2) we incurred the reduction in impurity. The proposed approach of gini index classifier is as follows in Algorithm 1:

Algorithm 1: skeleton of Gini Index classification

Gini_index(D,A).

1) If all records are having either same class labels or having same attribute values, then go to step 2 and STOP otherwise go to step 3.

2) Specify node as a leaf node; classify datasets D into the attribute set 'A'. And return leaf node.

3) Specify node as a root node and got to step 4.

4) Find the best split using equation (2) by selecting the best attribute having least value of GINI index calculated 
using equation (1) and calculate the GINI gain using equation (3).

5) Specify the possible outcomes of step 4 as ' $O$ '.

6) For each o $\in \mathrm{O}$ do step 7 to step

7) Traverse through the edge $\mathrm{E}$ according to the condition specified in the step 4.

8) Call Gini_index (E(o), 'A') as a child node

9) Add the child node as descendent node of root and label the edge (root->child) as o.

10) Return root.

TABLE II: DATASET FOR 30 FACIAL IMAGES

\begin{tabular}{|c|c|c|c|c|c|}
\hline $\begin{array}{c}\text { Openin } \\
\text { g of } \\
\text { eyes } \\
\text { oe }\end{array}$ & $\begin{array}{l}\text { Width of } \\
\text { eyes } \\
\text { we }\end{array}$ & $\begin{array}{l}\text { Heigh } \\
t \text { of } \\
\text { Eyebr } \\
\text { ows } \\
\text { he }\end{array}$ & $\begin{array}{l}\text { Opening of } \\
\text { mouth } \\
\text { om }\end{array}$ & $\begin{array}{c}\text { Width } \\
\text { of } \\
\text { mouth } \\
\text { wm }\end{array}$ & $\begin{array}{c}\text { Class_Labe } \\
1\end{array}$ \\
\hline 13.5 & 38.5 & $\begin{array}{c}19.63 \\
3\end{array}$ & 9.1677 & 27 & Neutral \\
\hline 14.5 & 36 & 21.8 & 9.1677 & 26 & Neutral \\
\hline 13.5 & 30 & $\begin{array}{c}22.81 \\
67\end{array}$ & 8.333 & 23 & Neutral \\
\hline 13.5 & 31.5 & $\begin{array}{c}24.13 \\
33\end{array}$ & 8.333 & 24 & Neutral \\
\hline 17 & 36.5 & 24.5 & 11.677 & 29 & Neutral \\
\hline 15 & 44.5 & $\begin{array}{c}26.31 \\
67\end{array}$ & 6.6667 & 31 & Happy \\
\hline 17.5 & 39 & $\begin{array}{c}30.03 \\
3\end{array}$ & 9.1667 & 26 & Happy \\
\hline 11.5 & 41.5 & $\begin{array}{c}23.51 \\
67\end{array}$ & 8.33 & 25 & Happy \\
\hline 21.5 & 44.5 & $\begin{array}{c}26.36 \\
67\end{array}$ & $\begin{array}{l}5.833 \\
\end{array}$ & 28 & Happy \\
\hline 18 & 37.5 & 23.25 & 9.16667 & 22 & Happy \\
\hline 25.5 & 32.5 & $\begin{array}{c}34.93 \\
33 \\
\end{array}$ & 9.16667 & 26 & Surprise \\
\hline 29 & 37.5 & $\begin{array}{c}40.61 \\
67 \\
\end{array}$ & 11.667 & 29 & Surprise \\
\hline 23.5 & 33 & $\begin{array}{c}34.33 \\
3\end{array}$ & 9.1667 & 28 & Surprise \\
\hline 29.5 & 32.5 & $\begin{array}{c}35.98 \\
33\end{array}$ & 12.5 & 24 & Surprise \\
\hline 22.5 & 32 & $\begin{array}{c}23.83 \\
3 \\
\end{array}$ & 11.667 & 25 & Surprise \\
\hline 15 & 34 & $\begin{array}{c}21.86 \\
67\end{array}$ & 10 & 28 & Sad \\
\hline 14 & 35.5 & $\begin{array}{c}23.53 \\
33 \\
\end{array}$ & 6.667 & 28 & Sad \\
\hline 13.5 & 37.5 & $\begin{array}{c}19.96 \\
67\end{array}$ & 5.8333 & 29 & Sad \\
\hline 13 & 30.5 & $\begin{array}{c}21.36 \\
67\end{array}$ & 7.5 & 24 & Sad \\
\hline 13 & 35.5 & $\begin{array}{c}20.86 \\
67\end{array}$ & 5.8333 & 28 & Sad \\
\hline 13.5 & 32 & $\begin{array}{c}19.53 \\
3\end{array}$ & 8.333 & 23 & Angree \\
\hline 14 & 35 & $\begin{array}{c}21.06 \\
67\end{array}$ & 8.333 & 30 & Angree \\
\hline 13 & 34.5 & $\begin{array}{c}16.76 \\
67\end{array}$ & 8.333 & 24 & Angree \\
\hline 13.5 & 33 & $\begin{array}{c}18.48 \\
33\end{array}$ & 7.5 & 26 & Angree \\
\hline 13 & 35 & $\begin{array}{c}17.41 \\
67\end{array}$ & 10.833 & 23 & Angree \\
\hline
\end{tabular}

Hence, in this way the tree is formed and best attributes are selected for best split. In our paper there are 30 data sets, 5 data sets for each six facial expressions and there are 5 attributes, i.e. 5 facial animation parameters. Table II, described the data set considered and Fig. 2 described the tree created using GINI Index considering the data set form Table II.

\section{Rule Extraction from Decision Tree}

Now the tree generated using above dataset is shown in fig 2. The decision tree is further converted in IF- Then rule for prediction of test dataset.

IF-THEN rules.

R1: IF oe $>20.5$ THEN: Class_Label = 'Surprise'

R2: IF oe $<=20.5$ AND he $<=20.67$ THEN

Class_label $=$ 'Angree'

R3: IF oe $<=20.5$ AND he $>20.67$ AND we $>37$ THEN: Class Label $=$ 'Happy'

R4: IF oe $<=20.5$ AND he $>20.67$ AND we $<=37$ AND $w m>26$ THEN Class_Label $=$ ' SAD'.

R5: IF oe $<=20.5$ AND he $>20.67$ AND we $<=37$ AND $w m<=26$ AND om $<9.5$ THEN Class_Label $=$ 'Neutral' R6: IF oe $<=20.5$ AND he $>20.67$ AND we $<=37$ AND wm $<26$ and om $>9.5$ THEN Class Label $=$ 'Fear' $\mathrm{R} 7: \mathrm{wm}<=26$ AND om $>9.5$ THEN Image is FEAR image.

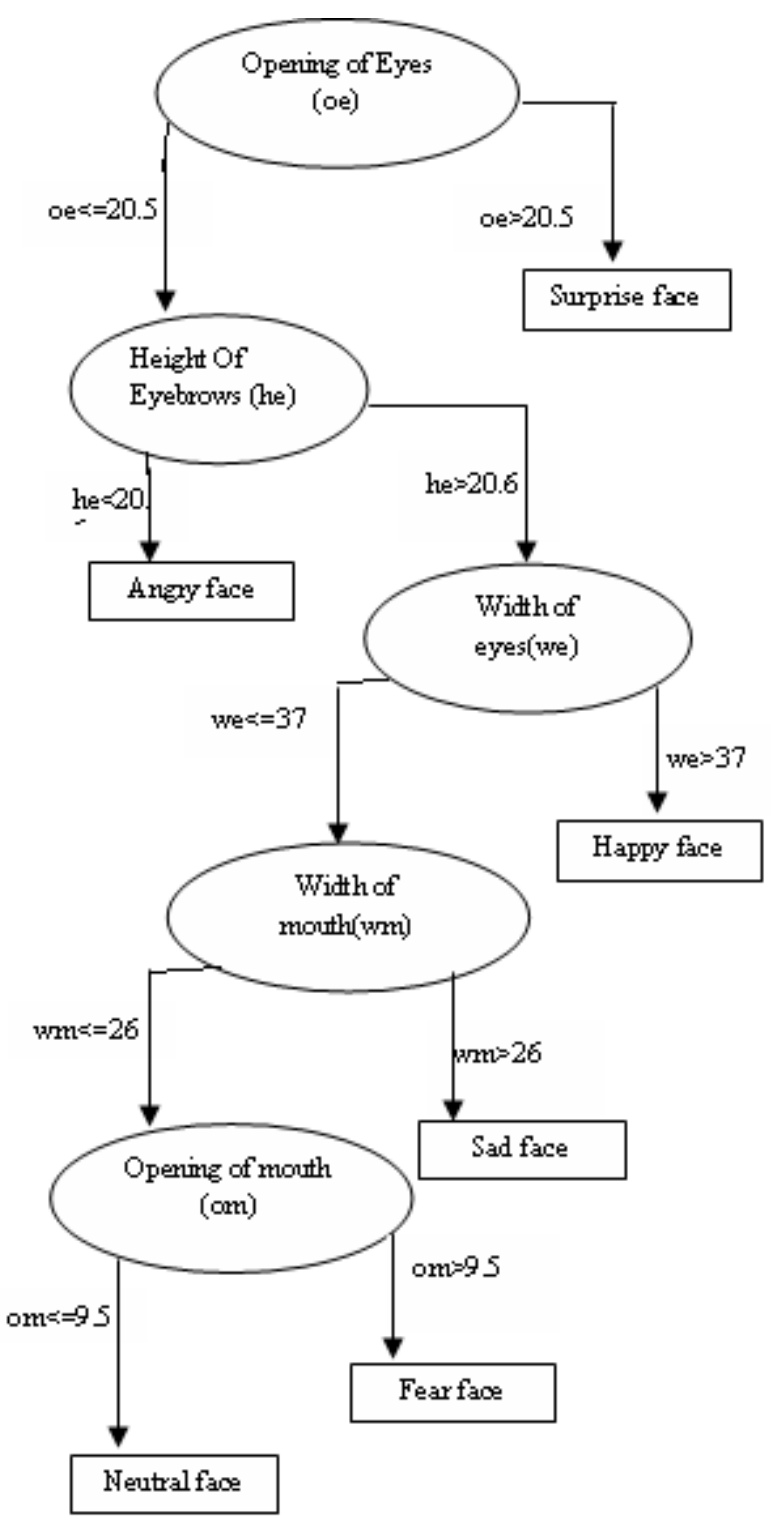

Fig. 2. Decision tree for classifying image using gini index. 


\section{EXPERIMENTAL RESULTS}

We applied the above specified methods to determine different facial expression on JAFFE database. In Jaffe data base 213 images are there. In this database 10 images and each image has 3-4 different expression images. We divided $2 / 3$ of dataset for training and $1 / 3$ dataset for testing. For preprocessing of image most of the image contain non-information part that are removed before going to process for template matching.

\section{CONCLUSION}

In the proposed paper we explain a novel approach for expression detection of person. As expression is one of the important parts of human being. In this paper we used the data mining statistical approach, using information gain and gini index in order to recognize the correct emotion. In experiment $80.9 \%$ of accuracy in order to recognize the correct image. In future we will try to improve the accuracy of algorithm conclusion section is not required. Although a conclusion may review the main points of the paper, do not replicate the abstract as the conclusion. A conclusion might elaborate on the importance of the work or suggest applications and extensions.

\section{ACKNOWLEDGMENT}

The authors wish to thank the organization [12] for providing Jeffe dataset.

\section{REFERENCES}

[1] P. Ekman and W. Friesen, Facial Action Coding System, Consulting Psychologists Press, 1977, ch 2, pp. 24-29.

[2] F. Kawakami, H. Yamada, S. Morishima, and H. Harashima, "Construction and psychological evaluation of 3-D emotion space," Biomedical Fuzzy and Human Sciences, vol. 1, no. 1, pp. 33-42, 1995.

[3] M. Rosenblum, Y. Yacoob, and L. S. Davis, "Human expression recognition from motion using a radial basis function network architecture," IEEE Trans. on Neural Networks, vol. 7, no. 5, pp. 1121-1138, Sept. 1996

[4] M. Pantic and L. J. M. Rothkrantz, "Automatic analysis of facial expressions: The state of the art," IEEE Trans. Pattern Analysis \& Machine Intelligence, vol. 22, no. 12, pp. 1424-1445, Dec. 2000.
[5] Y. S. Gao, M. K. H. Leung, S. C. Hui, and M. W. Tananda, "Facial expression recognition from line-based caricature," IEEE Trans. System, Man, \& Cybernetics (Part A), vol. 33, no. 3, pp. 407-412, May 2003.

[6] Y. Xiao, N. P. Chandrasiri, Y. Tadokoro, and M. Oda, "Recognition of facial expressions using 2-D DCT and neural network," Electronics and Communications in Japan, Part 3, vo. 82, no. 7, pp.1-11, July 1999.

[7] L. Ma and K. Khorasani, "Facial expression recognition using constructive feedforward neural networks," IEEE Trans. System, Man, and Cybernetics (Part B), vol. 34, no. 4, pp. 1588-1595, 2003.

[8] L. Ma, Y. Xiao, K. Khorasani, and R. Ward, "A new facial expression recognition technique using 2-D DCT and K-means algorithms," in Proc. ICIP 04, 2004, pp.1269-1272.

[9] Y.-W. Wu, H. Liu, and H.-B. Zha, "Modeling facial expression space for recognition," National Natural Science Foundation of China (NSFC). Project No: 60175025, P.R.China.

[10] J.-Y. Chang and J.-L. Chen, "A facial expression recognition system using neural networks", in Proc. IJCNN '99, pp. 3511-3516, vol. 5 1999.

[11] L. C. Jain, U. Halict, and I. Hayashi, Intelligent Biometric Techniques in Fingerprint and Face Recognition, CRC Press, 1999.

[12] Kasrl. [Online]. Available: www.kasrl.org/jaffe_download.html

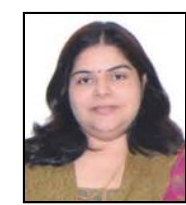

Priyanka Tripathi is an assistant professor in the department of Master of Computer Application at National Institute of Technology Raipur Chhattisgarh. She has completed her Ph.D. from MANIT Bhopal MP She has also worked with Tata Consultancy Services TCS. She is the Lifetime Member of The Computer Society of India. She is also a member of IEEE, ACM \& IACSIT.

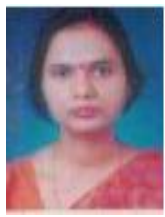

Kesari Verma completed her postgraduate in 1998 and got doctorate in 2007. She is current Assistant professor in National Institute of Technology, Raipur. She has 12 year R \& D and Teaching experience. She has more than 30 publications in journals and conferences.

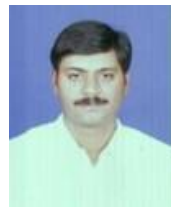

Ligendra Verma completed his postgraduate in 1998 from Govt. Engineering College Raipur. He has 10 year of teaching and 10 year of industries experience. He is pursuing his Ph.D. in Image Mining.

Nazil Parveen completed her B.E. from Guru Ghansidas University and M. Tech from National Institute of Technology, Raipur. 